\title{
Postural control in girls with adolescent idiopathic scoliosis while wearing a Chêneau brace or performing active self- correction: A pilot study
}

\author{
Elżbieta Piątek ${ }^{\text {Corresp., } 1}$, Michał Kuczyński ${ }^{2}$, Bożena Ostrowska ${ }^{1}$ \\ 1 Faculty of Physiotherapy, University School of Physical Education in Wroclaw, Wrocław, Poland \\ 2 Faculty of Physical Education and Physiotherapy, Opole University of Technology, Opole, Poland \\ Corresponding Author: Elżbieta Piątek \\ Email address: elzbieta.piatek90@gmail.com
}

Background. It is known that adolescent idiopathic scoliosis (AIS) is often accompanied by balance deficits. This reciprocal relationship must be taken into account when prescribing new therapeutic modalities because these may differently affect postural control, interacting with therapy and influencing its results. Objective. The purpose was to compare postural control in girls with AIS while wearing the Chêneau brace (BRA) or performing active self-correction (ASC) with their postural control in a quiet comfortable stance. Methods. Nine subjects were evaluated on a force plate in three series of two 20-s quiet standing trials with eyes open or closed; three blocks were randomly arranged: normal quiet stance (QST), quiet stance with BRA, and quiet stance with ASC. On the basis of centre-of-pressure (COP) recordings, the spatial and temporal COP parameters were computed. Results and Discussion. Performing ASC was associated with a significant backward excursion of the COP mean position with eyes open and closed ( $E S=0.56$ and 0.65 , respectively; $p<0.05$ ). This excursion was accompanied by an increase in the COP fractal dimension ( $E S=1.05$ and $0.98 ; p<0.05)$ and frequency $(E S=0.78 ; p=0.10$ and $E S=1.14 ; p<0.05)$ in the mediolateral $(M L)$ plane. Finally, both therapeutic modalities decreased COP sample entropy with eyes closed in the anteroposterior (AP) plane. Wearing BRA resulted in ES $=$ $1.45(p<0.05)$ while performing ASC in ES $=0.76(p=0.13)$. Conclusion. The observed changes in the fractal dimension (complexity) and frequency caused by ASC account for better adaptability of patients to environmental demands and for their adequate resources of available postural strategies in the $\mathrm{ML}$ plane. These changes in sway structure were accompanied by a significant (around $25 \mathrm{~mm}$ ) backward excursion of the mean COP position. However, this improvement was achieved at the cost of lower automaticity, i.e. higher attentional involvement in postural control in the AP plane. Wearing BRA may have an undesirable effect on some aspects of body balance. 


\section{Postural control in girls with adolescent idiopathic}

2 scoliosis while wearing a Chêneau brace or

3 performing active self-correction: A pilot study

4

5 Elżbieta Piątek ${ }^{1}$, Michał Kuczyński ${ }^{2}$, Bożena Ostrowska ${ }^{1}$

6

$7{ }^{1}$ Faculty of Physiotherapy, University School of Physical Education in Wroclaw, Wroclaw, 8 Poland

92 Faculty of Physical Education and Physiotherapy, Opole University of Technology, Opole, 10 Poland

11

12 Corresponding author:

Elżbieta Piątek, c/o Bożena Ostrowska

Paderewskiego 35, Wroclaw, 51-612, Poland

Email address: elzbieta.piatek90@gmail.com

\section{Abstract}

Background. It is known that adolescent idiopathic scoliosis (AIS) is often accompanied by balance deficits. This reciprocal relationship must be taken into account when prescribing new therapeutic modalities because these may differently affect postural control, interacting with therapy and influencing its results.

Objective. The purpose was to compare postural control in girls with AIS while wearing the Chêneau brace (BRA) or performing active self-correction (ASC) with their postural control in a quiet comfortable stance.

Methods. Nine subjects were evaluated on a force plate in three series of two 20 -s quiet standing trials with eyes open or closed; three blocks were randomly arranged: normal quiet stance (QST), quiet stance with BRA, and quiet stance with ASC. On the basis of centre-of-pressure (COP) recordings, the spatial and temporal COP parameters were computed. 
29 Results and Discussion. Performing ASC was associated with a significant backward excursion 30 of the COP mean position with eyes open and closed (ES $=0.56$ and 0.65 , respectively; $\mathrm{p}<$ 31 0.05). This excursion was accompanied by an increase in the COP fractal dimension (ES $=1.05$ 32 and $0.98 ; \mathrm{p}<0.05)$ and frequency $(\mathrm{ES}=0.78 ; \mathrm{p}=0.10$ and $\mathrm{ES}=1.14 ; \mathrm{p}<0.05)$ in the 33 mediolateral (ML) plane. Finally, both therapeutic modalities decreased COP sample entropy 34 with eyes closed in the anteroposterior (AP) plane. Wearing BRA resulted in ES $=1.45(\mathrm{p}<$ $350.05)$ while performing ASC in $\mathrm{ES}=0.76(\mathrm{p}=0.13)$.

36 Conclusion. The observed changes in the fractal dimension (complexity) and frequency caused 37 38 by ASC account for better adaptability of patients to environmental demands and for their adequate resources of available postural strategies in the ML plane. These changes in sway structure were accompanied by a significant (around $25 \mathrm{~mm}$ ) backward excursion of the mean COP position. However, this improvement was achieved at the cost of lower automaticity, i.e. higher attentional involvement in postural control in the AP plane. Wearing BRA may have an undesirable effect on some aspects of body balance.

\section{Introduction}

Adolescent idiopathic scoliosis (AIS) is one of the common spinal deformities observed during adolescence, affecting 2-4\% of individuals aged 10-16 years (Hawasli, Hullar \& Dorward, 2015). AIS can introduce wide-ranging dysfunction in important bodily systems and organs (Simoneau et al., 2006; Wang et al., 2011; Weinstein et al., 2008). Research in the recent decade has attempted to determine if AIS patients show proprioceptive and somatosensory impairment. Several studies on AIS prognosis have reported abnormal somatosensory function, balance control, and proprioception (Guo et al., 2006). Numerous researchers present that standing balance assessments show greater postural instability in AIS patients compared with agematched controls (Gaudreault et al., 2005; Karimi, Kavyani \& Kamali, 2016; Chow et al., 2006). The cited literature suggests that young patients with scoliosis are more susceptible than healthy individuals to external perturbations and have greater difficulty in righting the body after disturbed balance (Karimi, Kavyani \& Kamali, 2016).

The underlying cause of impaired postural control is considered multifactorial albeit strongly associated with vestibular dysfunction and defects in certain structures of the central nervous system (Hawasli, Hullar \& Dorward, 2015; Gauchard et al., 2001; Byl et al., 1997). As 
60 the medial vestibulospinal tract controls the axial muscles (Pialasse et al., 2013), changes in the

61 brain stem or sensorimotor cortex during the critical preadolescent and adolescent period of 62 growth can impair sensorimotor integration and therefore lead to inappropriate trunk muscle 63 activities, spine deformation, and greater instability. Hence, it is hypothesized that reduced 64 balance control in AIS patients may be caused by either impaired vestibular information transfer 65 66 67 68 69 70 71 72 73 74 75 76 77 78 79 80 or sensorimotor processing (Shi et al., 2013).

Two of the most common non-operative treatments prescribed for AIS are physiotherapy via active self-correction (ASC) and orthotic braces (Coillard et al., 2003; d'Amato, Griggs \& McCoy, 2001; Katz \& Durrani, 2001). ASC is a series of movements of realignment as a whole that the patients autonomously perform in order to reduce the scoliotic curves. This exercise has to be implemented as much as possible in three dimensions, which sometimes makes the ASC movement not easy to be understood and also not easy to be completed by the patient (Pizzetti et al., 2010). Bracing is generally recommended for skeletally immature patients with Cobb angles of $25-45^{\circ}$ so as to halt further progressive curvature and provide passive correction (Chow,

4 Leung \& Holmes, 2007). Within the group of rigid Thoraco-Lumbo-Sacral Orthoses (TLSO), the Chêneau brace (BRA) is most widely used in Poland (Zaborowska-Sapeta et al., 2011).

6 However, the clinical effects of bracing on postural balance in the AIS-afflicted population have only been addressed by a few studies, which report inconsistent findings. In the available literature, there is evidence that a bracing intervention can improve postural stability and balance in the sitting position (Smith \& Emans, 1992). One investigation confirmed that bracing did not impede stability or balance during upright stance on a solid surface but significantly increased the centre-of-pressure (COP) sway area, displacement, and mediolateral amplitude in patients standing on an unstable surface (Chow, Leung \& Holmes, 2007). Other studies have reported no significant clinical effects, among others, no changes in COP displacement or sway area, after a 4-month application of the Boston bracing system compared with controls (Sadeghi et al., 2008). Instead, greater postural stiffness in the anteroposterior direction and reduced mediolateral balance control were observed. Another study demonstrated improved postural balance after 4 months of bracing in young AIS patients (Paolucci et al., 2013; Khanali et al., 2015).

A significant issue is that the vast majority of studies have focused solely on the effects of BRA-induced alignment on postural control whereas few have addressed autocorrective-based approaches (three-dimensional ASC). The diagnostic and therapeutic recommendations 
91 published by the Society on Scoliosis Orthopaedic and Rehabilitation Treatment (SOSORT)

92 show consensus on the use of autocorrection exercises and the efficacy of this intervention 93 (Negrini et al., 2018). To the authors' knowledge, there are no studies that have evaluated the 94 effects of ASC (autocorrection). Therefore, the aim of this study was to compare postural control 95 in girls with AIS while wearing BRA or performing ASC with their postural control in a quiet 96 comfortable stance. We hypothesized better efficacy of ASC in supporting postural control.

98 Materials \& Methods

\section{Participants}

100 Nine post-menarche female AIS patients aged 11-16 years (age: $14 \pm 1.48$ years, weight: $47.71 \pm$ 101 $5.61 \mathrm{~kg}$, height: $161.5 \pm 8.81 \mathrm{~cm}$, Cobb angle: $35.6 \pm 8.9^{\circ}$ ) from a local therapeutic rehabilitation centre participated in the study. All subjects had normal vision. Individual patient characteristics including scoliotic curvature details are presented in Table 1. The inclusion criteria for the participants were diagnosis of AIS by an independent physician and receiving conservative treatment: BRA and physiotherapeutic scoliosis-specific exercises (PSSE) recommended by SOSORT (Negrini et al., 2018). Excluded were patients with history of spine surgery, musculoskeletal or neurological disease, or any spinal pathology not comorbid with AIS. All participants received brace treatment for a minimum of 2 months $(3.56 \pm 1.42$ months $)$ with a dosage of 20 hours/day; the brace was removed for personal hygiene, exercise, or delineated rest periods. Each BRA was custom designed for three-dimensional curve correction by an experienced orthotics specialist. Figures 1 and 2 illustrate an exemplary BRA used in the study. All patients knew their own ASC movements which reduced scoliotic curves. These included different types of movements: (1) controlled self-elongation, having regard to the sagittal plane; (2) correction of the primary curve in the frontal plane; (3) correction of contiguous curves in the frontal plane; (4) correction of the primary curve in the horizontal plane. Written informed consent was obtained from all participants and their parent(s) or legal guardian(s). The study goals, procedures, and methods were explained in full, and the subjects were informed that they could withdraw at any time. The study was approved by the Senate Research Ethics Committee at the University School of Physical Education in Wroclaw, Poland (the approval number: 35/2016). 
122 [Table 1]

123 [Figure 1]

124 [Figure 2]

125

\section{Methods}

127 All procedures were performed in laboratory conditions. Postural control was assessed barefoot 128 on a Kistler force platform (Kistler 9281CA, Winterthur, Switzerland). Two-dimensional 129 horizontal coordinates of the COP data were recorded for $20 \mathrm{~s}$ at a sampling frequency of 100 130 Hz. Three bipedal quiet standing trials were performed: (1) QST- normal quiet stance: standing 131 upright with a neutral and comfortable stance with arms relaxed at the sides; (2) ASC: standing 132 upright with autocorrection; on the 'correction' command, the participant performed ASC; and 133 (3) BRA: standing upright wearing BRA.

134 Each trial was performed in eyes-open and eyes-closed conditions. The foot position (5 $135 \mathrm{~cm}$ apart) was standardized on the surface to ensure repeatability across trials and participants.

136 Task order was counterbalanced with $1 \mathrm{~min}$ of rest provided after each trial in order to minimize 137 the effects of fatigue or hysteresis (Chow et al., 2007). The participants were instructed to stand 138 as motionless as possible. Data acquisition began when the subject signalled they were ready. 139 Relevant COP outcome measures were determined separately for the mediolateral (ML) and the 140 anteroposterior (AP) direction. These were:

- COP SD [mm] - standard deviation of COP displacement from mean COP;

- COP sway range $[\mathrm{mm}]$ - difference between the maximum and minimum value of the COP;

- COP mean $[\mathrm{mm}]$ - mean value of the COP position;

- COP mean velocity $[\mathrm{mm} / \mathrm{s}]$ - COP excursion divided by trial time;

- COP fractal dimension - a non-linear dynamic parameter of COP where the greater the fractal dimension, the better the postural system adapts to changes;

- COP sample entropy - a non-linear dynamic parameter of COP where greater entropy (higher COP irregularity) suggests less attentional resources devoted to balance maintenance (greater automaticity); 
152

153

154

155

156

157

158

159

160

161

162

163

164

165

166

167

168

169

170

171

172

173

174

175

176

177

178

179

180

181

182

- COP frequency $[\mathrm{Hz}]$ - frequency of COP, indicative of the involvement of the neural system in postural regulation.

\section{Statistical Analysis}

Data were processed with the Statistica 12.0 software package (StatSoft, USA). The data met the criteria of normal distribution for all parameters of COP measures. Thus, to evaluate the possible effects and interactions of vision (eyes open, eyes closed) and posture (quiet standing, ASC, BRA), a $2 \times 3$ repeated analysis of variance (ANOVA) was conducted for all parameters of the COP in the ML and the AP planes separately. To assess differences between the three groups, the post-hoc Fisher's least significant difference (LSD) test was used. The level of significance was set at $\mathrm{p}<0.05$. Effect size (ES) was calculated to determine the effects of ASC and BRA on postural control compared with quiet standing. An effect size of 0.3 is a small effect, 0.5 is a moderate effect, and 0.8 is a strong effect.

\section{Results}

In the AP plane, the COP mean position showed the main effect of posture $(\mathrm{F}[2,16]=3.73 ; \mathrm{p}=$ 0.0047). The LSD test pointed at a significant backward shift of the COP mean position in both eyes open $(p=0.004)$ and eyes closed $(p=0.0002)($ Fig. 3$)$. The traditional measures of the COP dispersion displayed the main effects of vision in the AP plane for COP variability $(\mathrm{F}[1,8]=$ $17.78 ; p=0.003)$, range $(F[1,8]=18.33 ; p=0.0003)$, and velocity $(F[1,8]=9.57 ; p=0.001)$.

Eyes closure increased the values of these three latter parameters. In addition, there was a vision $\times$ posture interaction $(F[2,16]=3.89 ; \mathrm{p}=0.042)$ for range only, which arose because brace differently affected this parameter: the range increased during eyes closed $(p=0.003)$ and remained unchanged during eyes open. The COP sample entropy in the AP plane displayed the main effect of vision $(F[1,8]=6.87 ; p=0.031)$, indicating lower values with eyes closed. Posthoc analysis revealed that using a brace resulted in a significantly lower $(\mathrm{p}=0.02)$ sample entropy during eyes closed (Fig. 4). Interestingly, there were no differences between ASC and quiet standing.

In the ML plane, the results of ANOVA proved the main effect of posture on the COP fractal dimension $(F[2,16]=6.05 ; \mathrm{p}=0.011)$ and sway frequency $(\mathrm{F}[2,16]=10.7 ; \mathrm{p}=0.001)$. The post-hoc LSD test indicated the highest values of the COP fractal dimension during ASC 
183 with eyes open $(\mathrm{p}=0.01)$ and eyes closed $(\mathrm{p}=0.03)$ compared with the brace and quiet standing 184 (Fig. 5). The post-hoc analysis of frequency did not reveal significant changes (Fig. 6).

185 The means (SD) of all dependent variables including effect size values over 0.5 are 186 shown in Table 2.

187

188 [Table 2]

189 [Figure 3]

190 [Figure 4]

191 [Figure 5]

192 [Figure 6]

193

194 Discussion

195 The purpose of this study was to compare postural control in girls with AIS while wearing BRA 196 or performing ASC with their postural control in a quiet comfortable stance. In view of the 197 fundamental difference between the two therapeutic approaches, i.e. BRA and ASC, we 198 hypothesized better efficacy of ASC in supporting postural control. This prediction was mainly 199 based on the expected releasing degrees of freedom and facilitating exploratory function by ASC 200 as opposed to biomechanical constraints imposed by the brace, rendering the hip and trunk joints 201 practically inflexible. On the other hand, releasing degrees of freedom has been suggested as an 202 important characteristic of motor learning, which is necessary when exploring solutions for a 203 novel task (Sternad, 2018).

204 To find the effects of the investigated postures on the possible preferential use of 205 different sensory afferents, we examined the subjects with the manipulation of visual input. Such an experimental protocol provides unique opportunities to reveal how sensory and motor signals

207 208 209

212 by some enhancement in sensory integration and internal representation of verticality. The latter 213 proposition is consistent with some other studies on AIS (Gauchard et al., 2000; Simoneau et al., 
214 2006; Catanzariti et al., 2014), as well as on stroke or vestibular pathology (Pérennou et al., 215 2008; Borel et al., 2008).

216 In contrast to the weak interactions, there were strong main effects of vision on several

217 postural indices. Eyes closure deteriorated postural performance, as evidenced by the increased 218 COP variability and mean velocity. The same manipulation decreased COP entropy, indicating 219 the need for more conscious and less automatic postural control with occluded vision.

220 Additionally, eyes closure resulted in an increased COP frequency, suggesting that a higher rate 221 of the exploratory sway function is required when some of the normally available sensory inputs 222 are suppressed. The results of these manipulations add to our understanding of the differing 223 effects of BRA and ASC on the COP parameters in patients with AIS.

224 With regard to the specific results of this study, aimed at comparing the role of BRA and 225 ASC in postural control, four findings seem of particular value. First, ASC resulted in a 226 significant backward shift of the COP mean position on a hard surface in both visual conditions. 227 This is equivalent to a very similar mean backward shift of the body centre of mass. Second, this 228 shift was accompanied by an increase in the ML fractal dimension and frequency. Third, both 229 therapeutic modalities increased sway amplitude only in the AP plane with eyes closed.

230 Furthermore, in the AP plane with eyes closed, sway entropy decreased with BRA and to a lesser 231 extent with ASC.

232 Mean COP position is seldom computed and analysed except in subjects standing at 233 heights (Carpenter et al., 2001) and in older persons (Jbabdi, Boissy \& Hamel, 2008), where fear 234 of falling significantly contributes to increasing the distance between the limits of stability and 235 the COP mean position. The fact of ignoring this index in the majority of studies probably 236 indicates a high consistency in adopting this position regardless of various sensory manipulations 237 and accounts for a presence of a strong set-point used by the central nervous system in 238 controlling erect stance. Therefore, in our subjects, this backward shift of the mean COP position 239 that resulted from the ASC posture must be interpreted as a relevant adjustment in postural 240 control. By virtue of the unexpected emergence and its impressive magnitude only, the backward 241 displacement of the COP cannot be instantly classified as a beneficial modification of posture.

242 Importantly, however, this modification was accompanied by an increase in the COP fractal 243 dimension and frequency, which manifest advantageous changes in the postural control system.

244 The fractal dimension is regarded as a measure of complexity of the investigated time-series and, 
245 within some limits, its higher values account for improvements in the overall postural strategies 246 and in the better adaptability to novel postural challenges, in particular. A higher COP

247 complexity reflects the ability of the central nervous system to use a variety of postural strategies 248 to maintain stable stance (Cone, Goble \& Rhea, 2017).

249 As documented by Cone, Goble \& Rhea (2017), balance training that focused at a better 250 sensory reweighting resulted in an increased complexity of sway. In the same vein, Casabona et 251 al. (2016) showed a larger fractal dimension in ballet dancers than in non-dancers and concluded 252 that this difference might indicate a rearrangement of sensory integration and motor adaptation 253 necessary to meet the particular demands of selected ballet performances. This is in line with our

254 255 256

257 258 259 260 261 262 263 264 265 266

267 268 269

270 271 272 273 274 275 understanding of the results of this study. Lower values of complexity found in AIS in habitual postures and while wearing braces may indicate the presence of biomechanical (and also psychological) constraints impeding the selection of sensorimotor strategies which might lead to optimal postural behaviour. The situation was quite different after the patients learned to adequately use the ASC posture. The mechanically and habitually imposed constraints were, at least partially, removed, as reflected by larger sway complexity, which indicated an improved activity of the sensorimotor system in the integrating available sensory inputs and of its capacity to perform the exploratory function.

It is important to point out that in contrast to the apparent gain in the organization of the ASC postural control in the ML plane, the respective changes in the AP plane revealed an unexpected outcome. While using ASC with eyes closed, our patients decreased sway entropy, which reflected more attention invested in postural corrections, characteristic of conscious or deliberate involvement into this process. By doing so, they constrained the normal automatic mode of operation and this interaction resulted in decreased postural performance, which was manifested by increases in sway variability. Although these changes in COP entropy are much lesser than those caused by BRA in the same condition, they may account for some problems that the AIS patients experienced in maintaining the backward shift associated with the new body alignment while performing ASC. Although these undesirable reactions occurred only in trials with eyes closed, when limited sensory input made postural control more challenging, their presence may be associated with a suboptimal design of the self-corrected posture or inadequate adherence of subjects to the rules established by therapists. Such questions have seldom, if ever, been asked in relation to postural performance of subjects with AIS. The results of this study 
276 suggest that a regular application of posturography in AIS patients may help answer these

277 questions and shed light on how to improve the treatment of this disease.

278 The main limitation of this pilot study is a small number of participants, which may have 279 concealed significant changes in postural performance brought about by BRA or ASC. Thus, in 280 addition to statistical significance, we also focused on the magnitude of effect sizes when 281 interpreting the study results. Nevertheless, the subsequent conclusions should be regarded with 282 caution unless confirmed by a replication study with an adequate sample size. The results of this 283 study may provide support as the input data for sample size calculation.

The second limitation concerns the lack of postural control assessment at some intermediate points between the ASC practice and bracing onset and the final test. The large backward shift of the mean COP position did not, most likely, occur during this final test but rather resulted from a continuous process of postural adjustments. One can only speculate that the latter modification of the gravity line alignment tends to optimize its position as a set-point for controlling erect stance. Owing to its direct relationship to poor posture in scoliosis, further research on this issue is warranted. Along the same lines, the investigation into the acute effects of new braces or postures on body stability may be helpful in clinical practice, facilitating the trade-off between body alignment and postural control.

\section{Conclusions}

295 This study compared postural control in girls with AIS while wearing BRA or performing ASC with their postural control in a quiet comfortable stance. We predicted better control during selfcorrection because its studious and diligent character seems to promote the exploratory function of postural sway, necessary in exploiting new strategies, as opposed to constraints imposed by the brace. Our hypothesis was confirmed by the increase in the COP complexity and frequency in the ML plane while performing active self-correcting movements in comparison with habitual standing. Such changes in complexity and frequency are supposed to account for better adaptability of patients to environmental demands and for their adequate resources of available postural strategies. However, this apparent improvement was achieved at the cost of lower automaticity, i.e. higher attentional involvement in postural control in the AP plane. Notably, the 
306

307

308

309

310

311

312

313

314

315

316

317

318

319

320

321

322

323

324

325

326

327

328

329

330

331

332

333

334

335

336

337

338

339

340

341

342

343

the mean COP position, which, we believe, may indicate a relevant adjustment in postural control.

\section{References}

1. Borel L, Lopez C, Péruch P, Lacour M. 2008. Vestibular syndrome: a change in internal spatial representation. Neurophysiologie Clinique/Clinical Neurophysiology, 38(6), 375 389.

2. Byl N, Holland S, Jurek A, Hu S. 1997. Postural imbalance and vibratory sensitivity in patients with idiopathic scoliosis: implications for treatment. Journal of Orthopaedic \& Sports Physical Therapy, 26(2), 60-68.

3. Carpenter MG, Frank JS, Silcher CP, Peysar GW. 2001. The influence of postural threat on the control of upright stance. Experimental Brain Research, 138(2), 210-218.

4. Casabona A, Leonardi G, Aimola E, La Grua G, Polizzi CM, Cioni M, Valle MS. 2016. Specificity of foot configuration during bipedal stance in ballet dancers. Gait \& Posture, 46, 91-97.

5. Catanzariti JF, Agnani O, Guyot MA, Wlodyka-Demaille S, Khenioui H, Donze C. 2014. Does adolescent idiopathic scoliosis relate to vestibular disorders? A systematic review. Annals of Physical and Rehabilitation Medicine, 57(6-7), 465-479.

6. Chow DH, Kwok ML, Cheng JC, Lao ML, Holmes AD, Au-Yang A, Wong MS. 2006. The effect of backpack weight on the standing posture and balance of schoolgirls with adolescent idiopathic scoliosis and normal controls. Gait \& Posture, 24(2), 173-181.

7. Chow DH, Leung DS, Holmes AD. 2007. The effects of load carriage and bracing on the balance of schoolgirls with adolescent idiopathic scoliosis. European Spine Journal, 16(9), 1351-1358.

8. Coillard C, Leroux MA, Zabjek KF, Rivard C. 2003. SpineCor-a non-rigid brace for the treatment of idiopathic scoliosis: post-treatment results. European Spine Journal, 12(2), 141-148.

9. Cone BL, Goble DJ,Rhea, CK. 2017. Relationship between changes in vestibular sensory reweighting and postural control complexity. Experimental Brain Research, 235(2), 547554.

10. d'Amato CR, Griggs S, McCoy B. 2001. Nighttime bracing with the Providence brace in adolescent girls with idiopathic scoliosis. Spine, 26(18), 2006-2012.

11. Gauchard GC, Lascombes P, Kuhnast M, Perrin PP. 2001. Influence of different types of progressive idiopathic scoliosis on static and dynamic postural control. Spine, 26(9), 1052-1058.

12. Gaudreault N, Arsenault AB, Larivière C, DeSerres SJ, Rivard CH. 2005. Assessment of the paraspinal muscles of subjects presenting an idiopathic scoliosis: an EMG pilot study. BMC Musculoskeletal Disorders, 6(1), 14. 
344

345

346

347

348

349

350

351

352

353

354

355

356

357

358

359

360

361

362

363

364

365

366

367

368

369

370

371

372

373

374

375

376

377

378

379

380

381

382

13. Guo X, Chau WW, Hui-Chan CW, Cheung CS, Tsang WW, Cheng JC. 2006. Balance control in adolescents with idiopathic scoliosis and disturbed somatosensory function. Spine, 31(14), E437-E440.

14. Hawasli AH, Hullar TE, Dorward IG. 2015. Idiopathic scoliosis and the vestibular system. European Spine Journal, 24(2), 227-233

15. Jbabdi M, Boissy P, Hamel M. 2008. Assessing control of postural stability in community-living older adults using performance-based limits of stability. $B M C$ Geriatrics, 8(1), 8.

16. Kabbaligere R, Lee BC, Layne CS. 2017. Balancing sensory inputs: Sensory reweighting of ankle proprioception and vision during a bipedal posture task. Gait \& Posture, 52, 244-250.

17. Karimi MT, Kavyani M, Kamali M. 2016. Balance and gait performance of scoliotic subjects: a review of the literature. Journal of Back and Musculoskeletal Rehabilitation, 29(3), 403-415.

18. Katz DE, Durrani AA. 2001. Factors that influence outcome in bracing large curves in patients with adolescent idiopathic scoliosis. Spine, 26(21), 2354-2361.

19. Khanali M, Arazpour M, Hutchins SW, Vahab Kashani R, Sadeghi H, Vahid Tari H. 2015. The influence of thoracolumbosacral orthoses on standing balance in patients with adolescent idiopathic scoliosis: a pilot study. Disability and Rehabilitation: Assistive Technology, 10(6), 452-457.

20. Negrini S, Donzelli S, Aulisa AG, Czaprowski D, Schreiber S, de Mauroy JC, Lebel A. 2018. 2016 SOSORT guidelines: orthopaedic and rehabilitation treatment of idiopathic scoliosis during growth. Scoliosis and Spinal Disorders, 13(1), 3.

21. Paillard T, Bizid R, Dupui P. 2007. Do sensorial manipulations affect subjects differently depending on their postural abilities? British Journal of Sports Medicine, 41(7), 435-438.

22. Paolucci T, Morone G, Di Cesare A, Grasso MR, Fusco A, Paulocci S, Iosa M. 2013. Effect of Chêneau brace on postural balance in adolescent idiopathic scoliosis: a pilot study. European Journal of Physical Rehabilitation Medicine, 49(5), 649-657.

23. Pérennou DA, Mazibrada G, Chauvineau V, Greenwood R, Rothwell J, Gresty MA, Bronstein AM. 2008. Lateropulsion, pushing and verticality perception in hemisphere stroke: a causal relationship? Brain, 131(9), 2401-2413.

24. Pialasse JP, Laurendeau S, Descarreaux M, Blouin J, Simoneau M. 2013. Is abnormal vestibulomotor responses related to idiopathic scoliosis onset or severity? Medical Hypotheses, 80(3), 234-236.

25. Pizzetti P, Romano M, Negrini A, Negrini S. 2010. Active self correction during different exercises and movements: a biomechanical study. Scoliosis, 5(S1), O22.

26. Rasman BG, Forbes PA, Tisserand R, Blouin JS. 2018. Sensorimotor manipulations of the balance control loop-beyond imposed external perturbations. Frontiers in Neurology, 9. 
383

384

385

386

387

388

389

390

391

392

393

394

395

396

397

398

399

400

401

402

403

404

405

406

407

408
27. Sadeghi H, Allard P, Barbier F, Chavet P, Gatto L, Rivard CH, Simoneau M. 2008. Bracing has no effect on standing balance in females with adolescent idiopathic scoliosis. Medical Science Monitor, 14(6), CR293-CR298.

28. Shi L, Wang D, Hui SC, Tong MC, Cheng JC, Chu WC. 2013. Volumetric changes in cerebellar regions in adolescent idiopathic scoliosis compared with healthy controls. The Spine Journal, 13(12), 1904-1911.

29. Simoneau M, Richer N, Mercier P, Allard P, Teasdale N. 2006. Sensory deprivation and balance control in idiopathic scoliosis adolescent. Experimental Brain Research, 170(4), 576-582.

30. Simoneau M, Richer N, Mercier P, Allard P, Teasdale N. 2006. Sensory deprivation and balance control in idiopathic scoliosis adolescent. Experimental Brain Research, 170(4), 576-582.

31. Smith RM, Emans JB. 1992. Sitting balance in spinal deformity. Spine, 17(9), $1103-$ 1109.

32. Sternad, D. 2018. It's not (only) the mean that matters: variability, noise and exploration in skill learning. Current opinion in behavioral sciences, 20, 183-195.

33. Wang WJ, Yeung HY, Chu WCW, Tang NLS, Lee KM, Qiu Y, Cheng JCY. 2011. Top theories for the etiopathogenesis of adolescent idiopathic scoliosis. Journal of Pediatric Orthopaedics, 31, S14-S27.

34. Weinstein SL, Dolan LA, Cheng JC, Danielsson A, Morcuende JA. 2008. Adolescent idiopathic scoliosis. The Lancet, 371(9623), 1527-1537.

35. Zaborowska-Sapeta K, Kowalski I M, Kotwicki T, Protasiewicz-Fałdowska H, Kiebzak W. 2011. Effectiveness of Cheneau brace treatment for idiopathic scoliosis: prospective study in 79 patients followed to skeletal maturity. Scoliosis, 6(1), 2. 


\section{Table 1 (on next page)}

Anthropometric and scoliotic curvature characteristics of the participants. 
1

No. Sex Age [years $] \quad$ Body mass $[\mathrm{kg}] \quad$ Body height $[\mathrm{cm}] \quad$ Risser sign $\quad$ AIS curve type $\quad$ Cobb angle $\left[{ }^{\circ}\right] \quad$

\begin{tabular}{|c|c|c|c|c|c|c|c|c|}
\hline 1 & Female & 14 & 45.6 & 163.5 & 3 & Right thoracic/left lumbar & $30 / 32$ & $\mathrm{~T} 8 / \mathrm{L} 2$ \\
\hline 2 & Female & 14 & 53.5 & 169 & 3.5 & Right thoracic/left lumbar & $28 / 40$ & $\mathrm{~T} 8 / \mathrm{L} 2$ \\
\hline 3 & Female & 12.5 & 52.2 & 163.5 & 3.5 & Right thoracic/left lumbar & $16 / 25$ & $\mathrm{~T} 7 / \mathrm{L} 1$ \\
\hline 4 & Female & 15 & 51.8 & 170.5 & 2 & Right thoracic/left lumbar & $18 / 35$ & $\mathrm{~T} 9 / \mathrm{L} 3$ \\
\hline 5 & Female & 15 & 53.1 & 171.5 & 3 & Right thoracic/left lumbar & $37 / 20$ & $\mathrm{~T} 8 / \mathrm{L} 1$ \\
\hline 6 & Female & 16 & 48.8 & 158 & 4 & Right thoracic/left lumbar & $32 / 30$ & $\mathrm{~T} 8 / \mathrm{L} 1$ \\
\hline 7 & Female & 14.5 & 40.8 & 153 & 1.5 & Right thoracic/left lumbar & $55 / 20$ & $\mathrm{~T} 7 / \mathrm{L} 1$ \\
\hline 8 & Female & 11 & 38 & 144.5 & 1 & Right thoracic/left lumbar & $26 / 20$ & $\mathrm{~T} 8 / \mathrm{L} 1$ \\
\hline 9 & Female & 14 & 45.6 & 160 & 3 & Right thoracic/left lumbar & $38 / 18$ & T9/L3 \\
\hline
\end{tabular}


Table 2 (on next page)

Mean $\pm \mathrm{SD}$ in $\mathrm{AP}$ and ML COP outcome measures for posture trials

* significant difference at $p<0.05$

$\dagger \mathrm{ES}>0.5 ; \dagger+\mathrm{ES}>0.8$ 
1

2

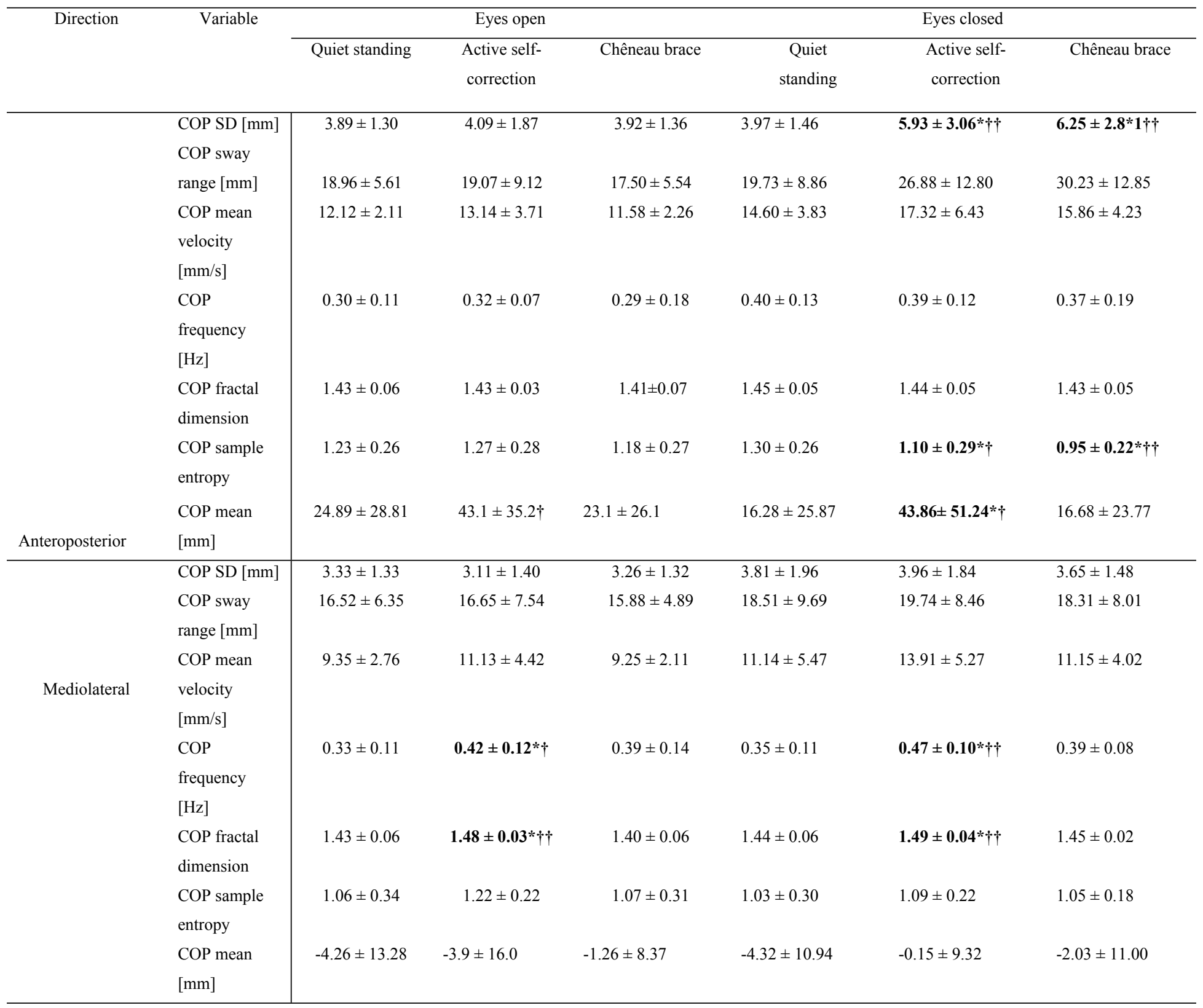


Figure 1

The Chêneau brace used in this study (back). 


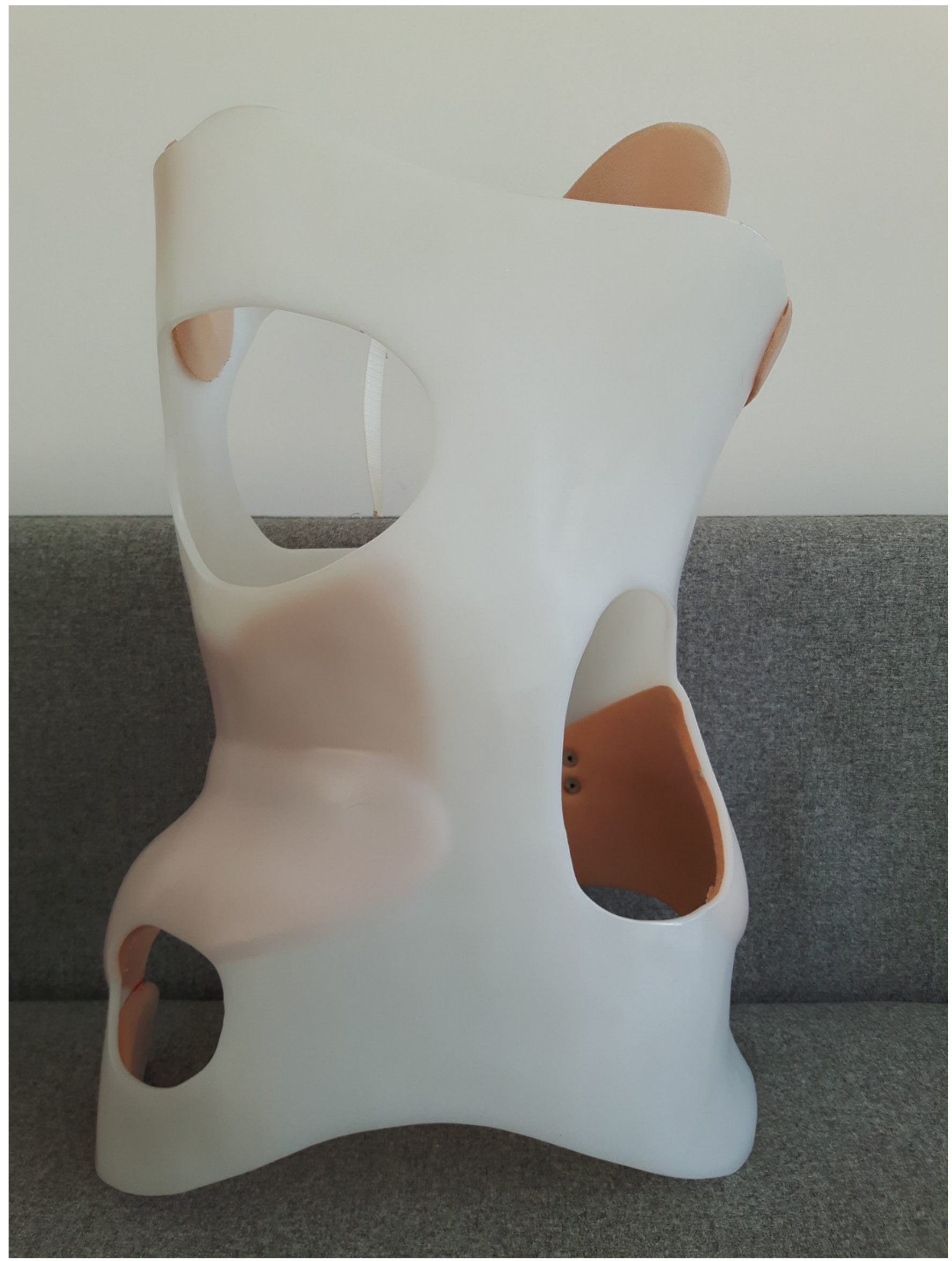

PeerJ reviewing PDF | (2019:03:36145:1:1:NEW 6 Jul 2019) 
Figure 2

The Chêneau brace used in this study (front). 


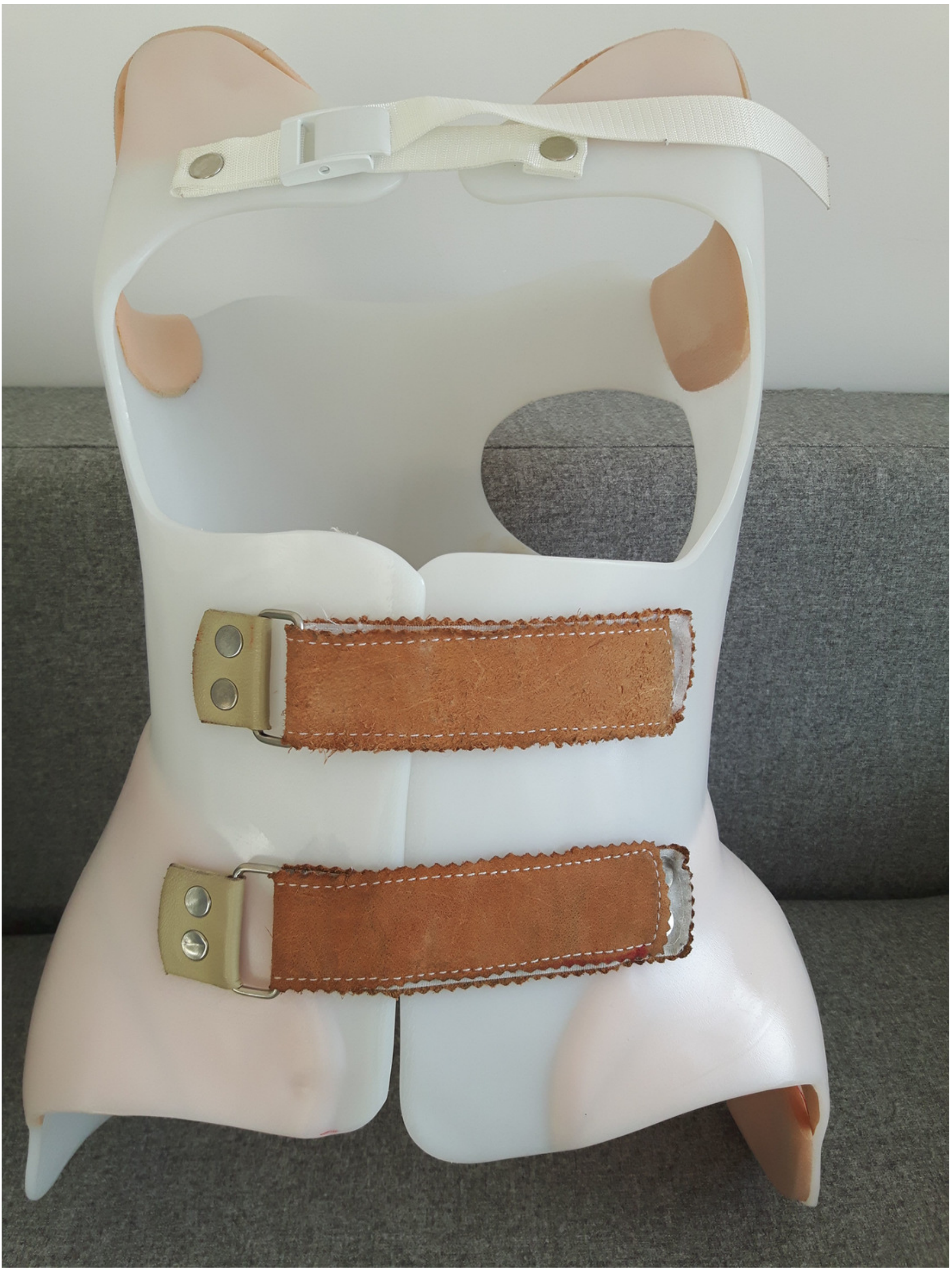


Figure 3 (on next page)

Mean (SD) values of COP mean position in the AP plane in the backward directions.

QST- quiet standing; ASC- active self-correction; BRA- Chêneau brace. White column- Eyes open; Shaded column- Eyes closed. Asterisks indicate a significant $(p<0.05)$ post hoc difference with respect to QST. 


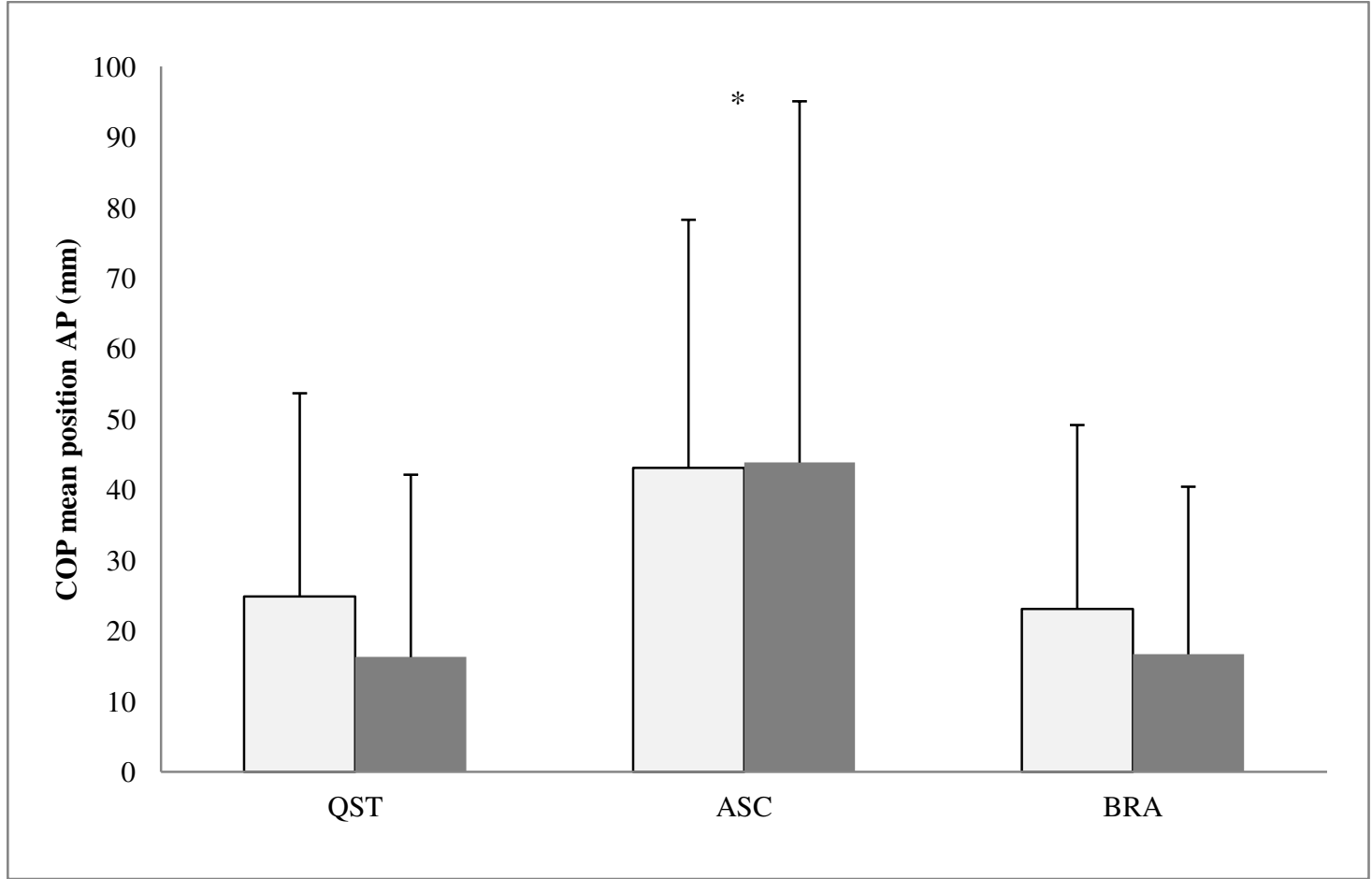


Figure 4 (on next page)

Mean (SD) values of Samle entropy in the AP plane.

QST- quite standing; ASC- active self-correction; BRA- Chêneau brace. White column- Eyes open; Shaded column- Eyes closed. Asterisks indicate a significant $(p<0.05)$ post hoc difference with respect to QST 


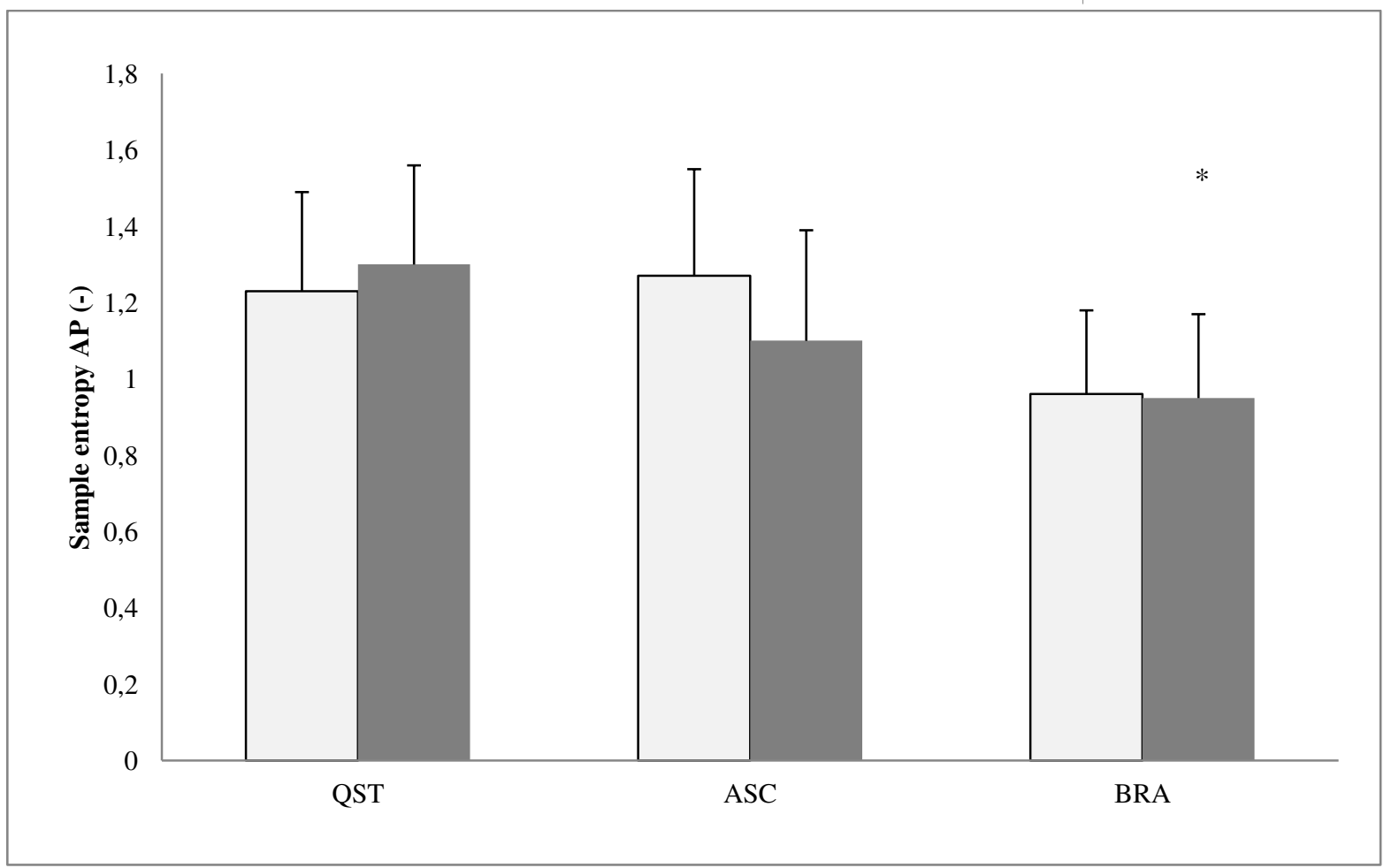


Figure $\mathbf{5}$ (on next page)

Mean (SD) values of COP fractal dimension in the ML plane.

QST- quiet standing; ASC- active self-correction; BRA- Chêneau brace. White column- Eyes open; Shaded column-Eyes closed. Asterisks indicate a significant $(p<0.05)$ post hoc difference with respect to QST 


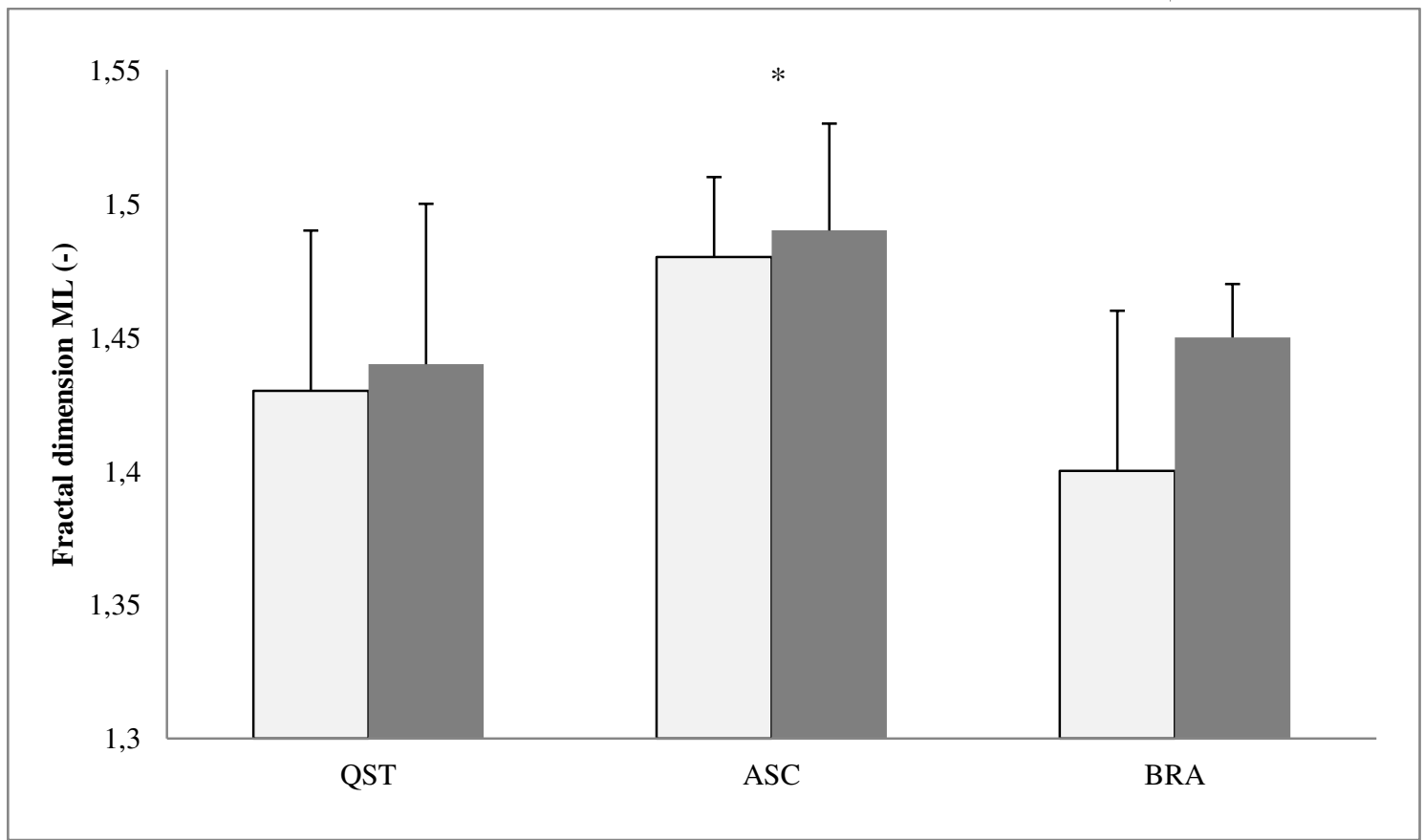


Figure 6 (on next page)

Mean (SD) values of COP frequency in the ML plane.

QST- quite standing; ASC- active self-correction; BRA- Chêneau brace. White column- Eyes open; Shaded column- Eyes closed. 


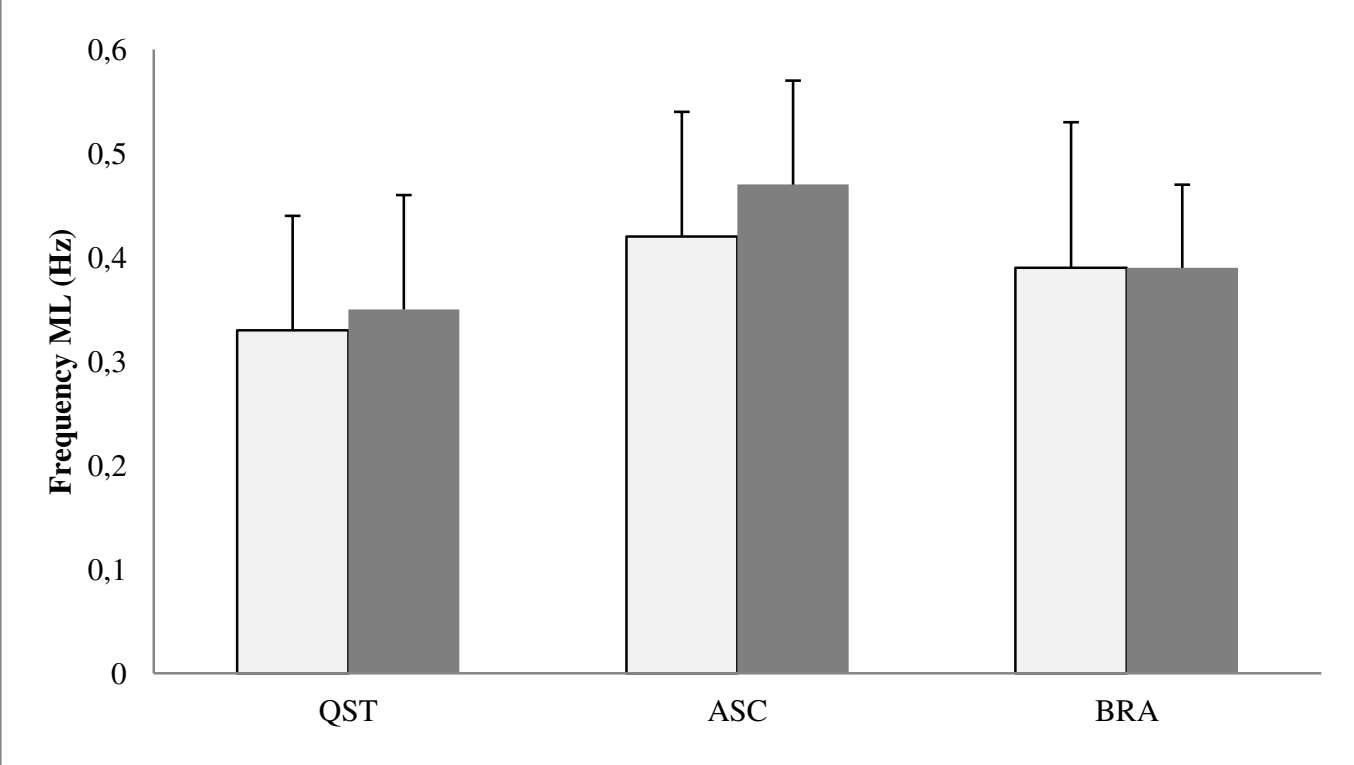

